\title{
TRATAMIENTO CON TERAPIA FOTODINÁMICA DE DOS CASOS DE EPITELIOPATÍA PIGMENTARIA RETINIANA DIFUSA
}

\section{TREATMENT OF TWO CASES OF DIFFUSE RETINAL PIGMENT EPITHELIOPATHY WITH PHOTODYNAMIC THERAPY}

\author{
ARMADÁ $\mathrm{F}^{1}$, ROMERO R ${ }^{2}$, ORTEGA I ${ }^{2}$, FONSECA A ${ }^{2}$
}

\begin{abstract}
RESUMEN
Caso clínico: Presentamos dos pacientes con epiteliopatía pigmentaria retiniana difusa (EPRD) tratados mediante terapia fotodinámica (TFD) con verteporfina.

La primera paciente fue tratada con TFD por tener afectación foveal. El segundo paciente recibió TFD por presentar EPRD recidivante con mala evolución a pesar de fotocoagulación con láser argón.

En ambos casos se resolvieron las lesiones y mejoró la agudeza visual. Tras once meses de seguimiento no se han producido recidivas.

Discusión: Los resultados obtenidos en nuestros pacientes con TFD son satisfactorios. Consideramos que es una opción terapéutica segura y eficaz, aunque son necesarios estudios aleatorizados a largo plazo.
\end{abstract}

Palabras clave: Epiteliopatía pigmentaria retiniana difusa, terapia fotodinámica, verteporfina, coriorretinopatía serosa central, lesiones exudativas multifocales.

\begin{abstract}
Case report: We present two patients with Diffuse Retinal Pigment Epitheliopathy (DRPE) treated with verteporfin photodynamic therapy (PDT). The first patient was treated with PDT because there was foveal involvement. The second patient received PDT because he had multiple recurrences of DRPE with poor resolution following previous argon-laser photocoagulation. Resolution of the lesions occurred in both cases with improvement in visual acuity. No recurrences have been documented at eleven months of follow-up.

Discussion: The outcomes in our patients were satisfactory. We consider PDT is a safe and effective option, but further randomized studies are necessary to confirm this (Arch Soc Esp Oftalmol 2006; 81: 603-606).

Key words: Diffuse retinal pigment epitheliopathy, photodynamic therapy, verteporfin, central serous chorioretinopathy, multifocal exudative lesions.
\end{abstract}

Recibido: 15/2/06. Aceptado: 19/10/06.

Servicio de Oftalmología. Hospital Universitario La Paz. Madrid. España.

1 Doctor en Medicina.

2 Licenciado en Medicina.

Comunicación presentada en el LXXXI Congreso de la S.E.O. (Zaragoza 2005).

Correspondencia:

Ricardo Romero Martín

Urbanización Quinta del Sol, 32

28231 Las Rozas (Madrid)

España

E-mail: romeromartinricardo@hotmail.com 


\section{INTRODUCCIÓN}

La epiteliopatía pigmentaria retiniana difusa (EPRD) fue descrita a principios de la década de los 70 (1). Esta entidad es considerada una variante atípica y severa de la coriorretinopatía serosa central (CSC) caracterizada por lesiones exudativas multifocales en el polo posterior (destacando la localización parapapilar temporal superior), tractos de atrofia del epitelio pigmentario de la retina (EPR) extendiéndose hacia la periferia inferior, y desprendimientos de retina no regmatógenos periféricos en casos graves (2).

La etiología de la EPRD es desconocida. Los datos obtenidos de las angiografías con verde indocianina demuestran que el fenómeno primario es una hiperpermeabilidad coroidea, afectándose el EPR de manera secundaria (3).

Presentamos dos pacientes con EPRD tratados mediante terapia fotodinámica (TFD) con verteporfina. Hemos realizado una búsqueda bibliográfica en MEDLINE encontrando un único caso publicado de EPRD tratada con TFD (4).

\section{CASOS CLÍNICOS}

Mujer de 49 años de edad que acude por disminución de agudeza visual (AV) del ojo izquierdo (OI) de 2 semanas de evolución. No presentaba antecedentes sistémicos ni oftalmológicos de interés. La agudeza visual corregida $(\mathrm{AVc})$ era de 1 en el ojo derecho (OD) y de 0,3 en el OI. El polo anterior de ambos ojos (AO) era normal. No se encontraron alteraciones en la retina del OD. En la exploración del fondo de ojo del OI se objetivaron múltiples focos exudativos en polo posterior que provocaban desprendimientos neurosensoriales serosos con afectación foveal. La angiografía con fluoresceína (AFG) de dicho ojo mostraba hiperfluorescencia precoz a nivel de los focos exudativos que aumentaba en intensidad y tamaño en las fases tardías del angiograma. En las sucesivas revisiones se observó mayor acúmulo de líquido subretiniano a nivel macular con empeoramiento de la AV del OI. Se optó por tratar con TFD con verteporfina aquellos focos activos identificados en la AFG; en una primera sesión se trató el foco responsable de la afectación foveal (fig. 1) y en una segunda sesión las lesiones más periféricas (fig. 2). Se utilizaron los parámetros del estudio TAP-Treatment of Age-rela-

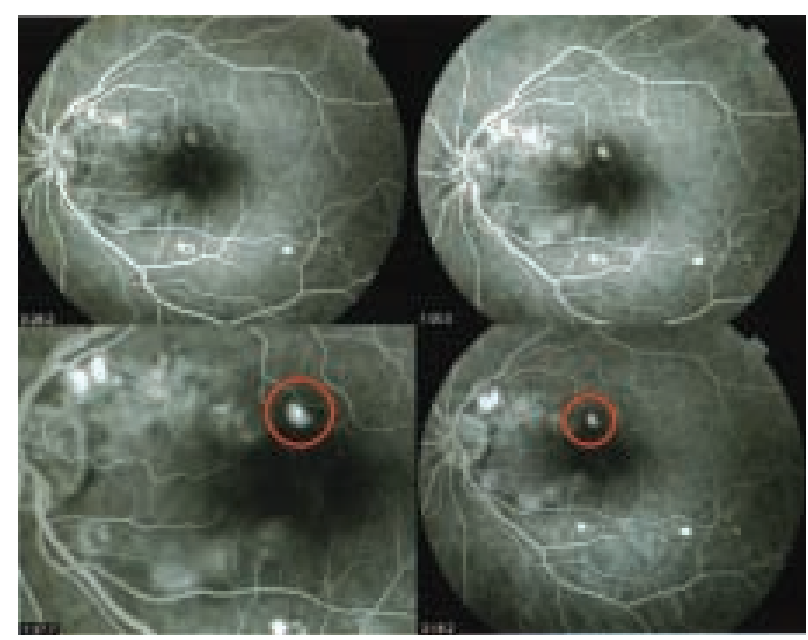

Fig. 1: Caso 1: Angiografía con fluoresceína del OI. Foco responsable de la afectación foveal a tratar con TFD.

ted macular degeneration with Photodynamic therapy (dosis lumínica: $50 \mathrm{~J} / \mathrm{cm}^{2}$; intensidad: 600 $\mathrm{mW} / \mathrm{cm}^{2}$; duración: 83 segundos; luz láser no térmica de $689 \mathrm{~nm}$ de longitud de onda) a excepción del tamaño del spot que se ajustó al tamaño de la lesión.

Pasado un mes de la última sesión de TFD se observó una resolución completa clínica y angiográfica de las lesiones exudativas (fig. 3). La AV del OI mejoró a 0,6 cuatro meses después de haber recibido el tratamiento y tras un seguimiento de 11 meses no se han producido recidivas y la $\mathrm{AV}$ ha evolucionado a la unidad.

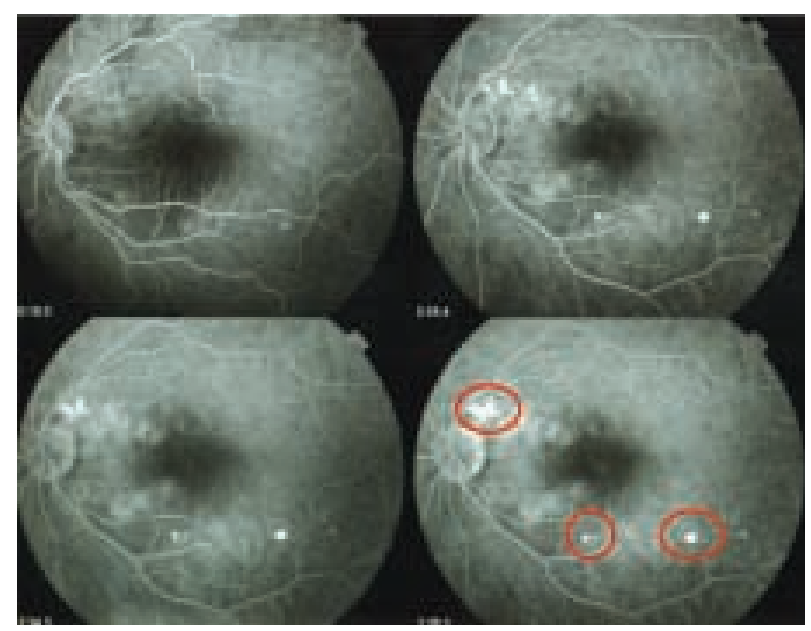

Fig. 2: Caso 1: Angiografía con fluoresceína del OI. Focos a tratar con TFD (el foco responsable de la afectación foveal ya ha sido tratado con TFD). 


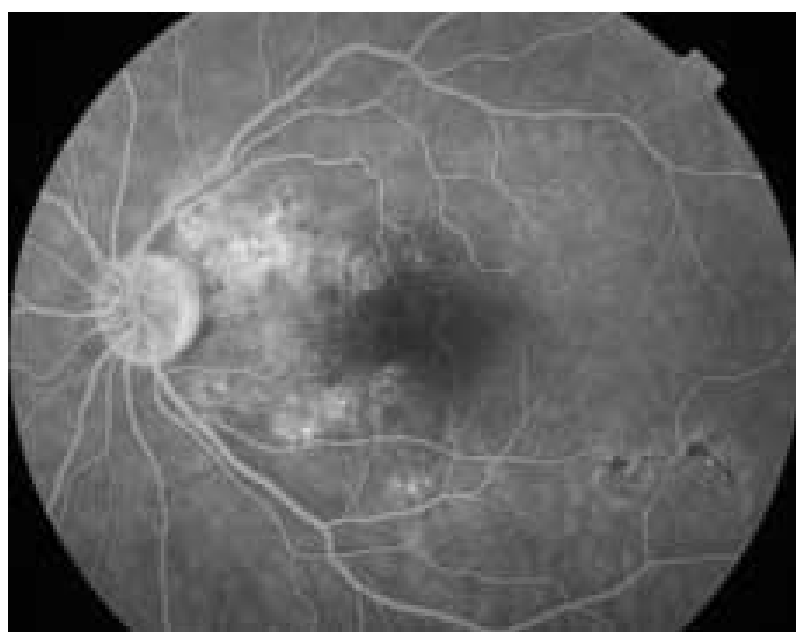

Fig. 3: Caso 1: Angiografía con fluoresceína del OI pasado un mes de la última sesión de TFD.

El segundo caso clínico es un varón de 42 años de edad que es remitido a nuestro centro con el diagnóstico de EPRD recurrente AO para valoración. El paciente había presentado múltiples brotes en $\mathrm{AO}$ desde 1992 tratados mediante fotocoagulación con láser Argón. A pesar del tratamiento, el OD presentaba un desprendimiento retiniano exudativo del polo posterior con focos activos crónicos y alteraciones del EPR a nivel foveal (fig. 4) con una AV de cuenta dedos.

En el OI la AVc era de 0,7. El polo anterior no presentaba hallazgos patológicos. El FO del OI revelaba lesiones multifocales exudativas en polo posterior y ecuador; la mácula estaba respetada pero presentaba alteraciones leves del EPR. En sector inferior de la retina se observaban los característicos tractos atróficos del EPR. Al realizar la AFG se apreciaba hiperfluorescencia desde tiempos iniciales en relación con los focos activos, que aumentaba en tamaño e intensidad en los tiempos tardíos (fig. 5).

Debido a las múltiples recidivas y a la mala evolución del ojo adelfo se decidió realizar TFD con

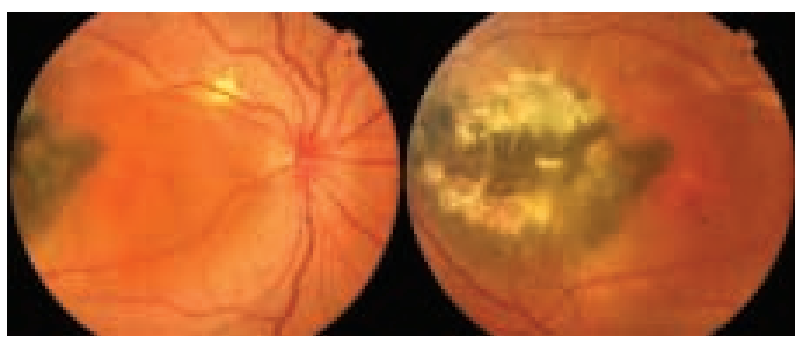

Fig. 4: Caso 2: Retinografía del OD.

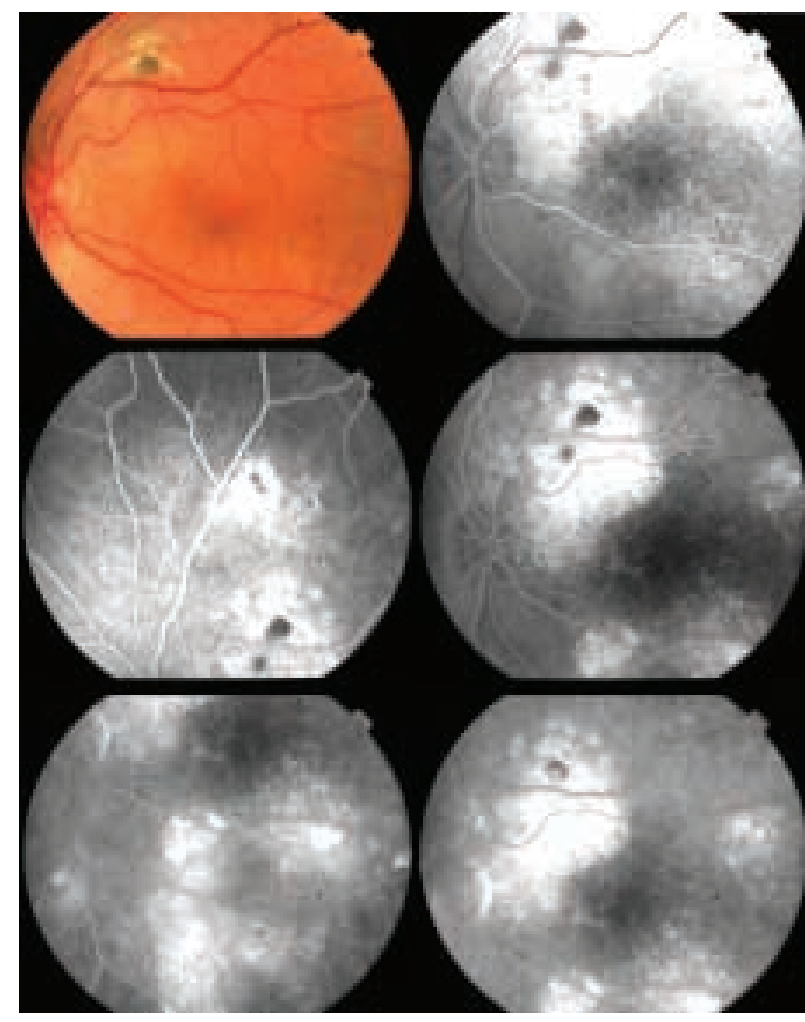

Fig. 5: Caso 2: Retinografía y angiografía con fluoresceína del OI previo al tratamiento con TFD.

verteporfina en el OI tratando en una única sesión todos los focos detectados con la AFG. Se utilizaron los mismos parámetros que en el caso anterior.

Pasados 11 meses desde el tratamiento la AV permanece estable y los focos inactivos (fig. 6).

\section{DISCUSIÓN}

La fotocoagulación con láser Argón ha demostrado ser eficaz en la resolución anatómica de las lesiones de la EPRD pero presenta una serie de inconvenientes: está contraindicada en focos subfoveales, puede provocar escotomas, no previene las recurrencias, y lo que es mas importante, no es concluyente que pueda mejorar, de manera estadísticamente significativa, la AV a largo plazo $(2,5)$.

Las ventajas de la TFD serían: se puede usar en focos subfoveales, al producir menor daño retiniano el riesgo de escotoma es menor, y se puede considerar un tratamiento fisiopatológico; la hipoperfusión coroidea inducida por la TFD contrarrestaría la hipermeabilidad de la coroides que como hemos 


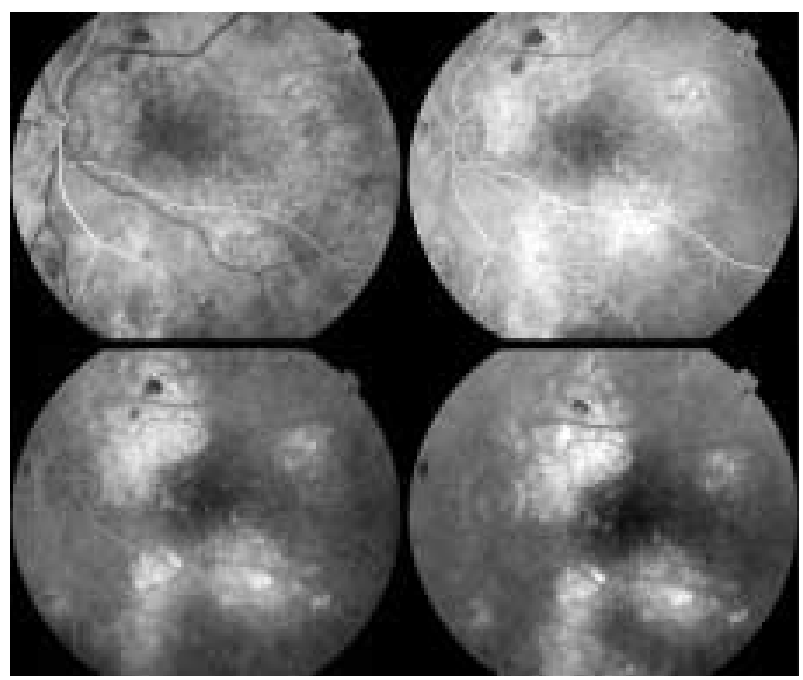

Fig. 6: Caso 2: Angiografía con fluoresceína del OI tras tratamiento con TFD.

comentado sería el trastorno primario de esta enfermedad. Dadas las características de este estudio, no podemos afirmar que la TFD mejore la $\mathrm{AV}$ final o prevenga las recurrencias a largo plazo.

Aunque existen varios casos descritos de CSC tratados con TFD, hasta la fecha solo hemos hallado un caso publicado en la literatura de EPRD tratada mediante TFD (4), con buenos resultados anatómicos y funcionales. Definimos la EPRD como una variante atípica de la CSC caracterizada por: lesiones exudativas multifocales en el polo posterior, tractos de atrofia del epitelio pigmentario de la retina, y desprendimientos de retina no regmatógenos periféricos en casos graves. Los resultado obtenidos con TFD en nuestros dos pacientes son satisfactorios. Consideramos que se trata de una opción terapéutica eficaz y segura, aunque son necesarios estudios aleatorizados a largo plazo.

\section{BIBLIOGRAFÍA}

1. Gass JD. Bullous retinal detachment. An unusual manifestation of idiopathic central serous choroidopathy. Am J Ophthalmol 1973; 75: 810-821.

2. Otsuka S, Ohba N, Nakao K. A long-term follow-up study of severe variant of central serous chorioretionpathy. Retina 2002; 22: 25-32.

3. Uyama M, Matsunaga H, Matsubara T, Fukushima I, Takahashi $K$, Nishimura T. Indocyanine green angiography and pathophysiology of multifocal posterior pigment epitheliopathy. Retina 1999; 19: 12-21.

4. Canakis C, Livir-Rallatos C, Panayiotis Z, Livir-Rallatos $G$, Persidis E, Conway MD, et al. Ocular photodynamic therapy for serous macular detachment in the diffuse retinal pigment epitheliopathy variant of idiopathic central serous chorioretinopathy. Am J Ophthalmol 2003; 136: 750-752.

5. Yanuzzi LA, Slakter JS, Kaufman SR, Gupta K. Laser treatment of diffuse retinal pigment epitheliopathy. Eur $J$ Ophthalmol 1992; 2: 103-114. 